\title{
Diotic and dichotic frequency recognition in a single-interval temporal interference procedure
}

\author{
WILLIAM A. YOST and GERALD B. THOMAS \\ Institute for Advanced Study of the Communication Processes and Psychology Department, \\ University of Florida, Gainesville, Florida 32611
}

\begin{abstract}
The frequency discriminability of a 70-dB SPL, 20-msec test tone followed 5 msec later by an equally intense $500-\mathrm{msec}, 800-\mathrm{Hz}$ interference tone was studied in a single-interval procedure for one diotic condition and three dichotic conditions. The test tone (T) and interference tone (I) were presented the same to both ears (ToIo, diotic condition) and in three dichotic conditions: (1) the interference tone was presented to the right ear $(R)$ and the test tone to the left ear $(L)\left(T_{L} I_{R}\right),(2)$ the interference tone was presented the same to both ears and the test tone to one ear (TmIo), and (3) the interference tone was presented the same to both ears and the test tone to both ears with an interaural phase reversal ( $\mathrm{T} \pi \mathrm{I}$ ). The threshold value for test tone frequency discrimination in the diotic temporal interference condition was approximately six times greater than that obtained without an interference tone. The three dichotic temporal interference conditions yielded essentially equivalent threshold values which were approximately 2.4 times that obtained when the test tone was presented without an interference tone. Therefore, although never equaling interference-free conditions, dichotic test tone presentations can improve frequency recognition relative to diotic conditions at intensities well above threshold. It is postulated that this improvement may be due to the spatial separation of test and interfering tones, rather than to possible differences in diotic vs. dichotic subjective intensities. Dichotic-diotic frequency recognition differences did not occur when a 100 -msec interval separated the test and interfering tone or when the interfering tone preceded the test tone.
\end{abstract}

Investigators have shown that the frequency recognition or discrimination of a test tone can be degraded if the test tone is followed closely in time by an interference tone [see Massaro (1975) and Yost, Berg, and Thomas (1976) for reviews of this literature]. In a recent paper, we (Yost, Berg, and Thomas, 1976) showed that, for highly trained observers and for situations involving minimal stimulus uncertainty, the amount of frequency recognition degradation achieved in this backward interference task is greatest in a single-interval psychophysical procedure. Little or no change in frequency discrimination over that obtained for the test tone in the absence of the interference tone is found for multipleinterval psychophysical procedures (two-alternative, temporal forced choice; same-different; and method of adjustment procedures).

Cudahy and Leshowitz (1974), Leshowitz and Cudahy (1973), Massaro (1970), and Sparks (1976) have shown different degrees of backward interference of test-tone frequency discrimination in a dichotic condition in which the test tone is presented to one ear and the interference tone is presented to

This research was supported in part by an NIH program project grant (NS 06459), an NIH training grant (NSO 5474), and an NIH research grant (NS 11157). the other ear. That is, there appears to be some increase in thresholds for frequency discrimination in this dichotic case over that obtained without an interfering tone. These results generally indicate, however, that the amount of threshold change is not as great as in the diotic case in which the test tone and interference tone are presented the same to both ears.

In the Yost, Berg, and Thomas (1976) paper, we showed that the amount of interference was dependent both on practice and on psychophysical procedure. We also argued that introduction of stimulus uncertainty could change the amount of frequency discriminability in temporal interference tasks. Since differences in amount of practice, psychophysical procedure, and amount of stimulus uncertainty exist among the procedures used by Cudahy and Leshowitz (1974), Leshowitz and Cudahy (1973), Massaro (1970), and Sparks (1976), we wished to determine the amount of backward interference that would exist for dichotic stimuli presented in the single-interval task with highly trained observers in cases involving minimal stimulus uncertainty. We were further interested in determining whether the amount of backward interference would depend on the way in which we presented the test tone or interference tone to the ears. Thus we tested for backward interference of frequency discrimination in four conditions: ${ }^{1}$ (1) the diotic case 
(To Io) in which the test tone (T) and interference tone (I) were presented the same (o) to both ears, (2) the $T_{L} I_{R}$ dichotic case in which the test tone was presented to the left ear (L) and the interference tone to the right ear (R), (3) the TmIo dichotic case in which the test tone was presented to one ear $(\mathrm{m})$ and the interference tone the same to both ears, and (4) the TrIo dichotic case in which the test tone was presented with an interaural phase reversal $(\pi)$ and the interference tone was presented the same to both ears.

\section{EXPERIMENT 1}

\section{Method}

Subjects. Two subjects (S1, S2), with normal hearing, who had participated in other psychoacoustical studies worked for approximately $30 \mathrm{~h}$ during this experiment.

Stimuli. In all cases, the test tone was a $20-\mathrm{msec}$ sinusoid and the interference tone a $800-\mathrm{Hz}, 500-\mathrm{msec}$ sinusoid. Both the test and interference tones were gated on and off at a zero crossing and no further filtering or temporal shaping was provided. The test tone terminated $5 \mathrm{msec}$ before the interfering tone began. Both tones were presented at $70 \mathrm{~dB}$ SPL, and the frequency of the test tone was either $800 \mathrm{~Hz}+\Delta \mathrm{f} / 2$ or $800 \mathrm{~Hz}$ $-\Delta f / 2$. That is, the frequency of the test tone was always varied in frequency symmetrical about $800 \mathrm{~Hz}$ (the frequency of the interference tone). The diotic condition was Tolo and the dichotic conditions were $T_{L} I_{R}$, TmIo, and TrIo (see introduction). ${ }^{2}$ The discriminability of the test tone was also measured when there was no interference tone (the NI condition).

A single-interval procedure was used in which on half the trials (determined randomly) the lower frequency test tone was presented and on the other half of the trials the higher frequency test tone was presented. The subject responded that the pitch of the test tone was either "high" or "low." Data were collected in 50-trial blocks, and the overall procedure was the same as that used by Yost, Berg, and Thomas (1976). The threshold value of $\Delta \mathbf{f}$ was determined by obtaining a two-point psychometric function of d' vs. $\Delta f$ (see Green \& Swets, 1974). At least 300 trials were used for each of the two points on the psychometric function to estimate the threshold value of $\Delta f$. Data were not collected until the value of $d^{\prime}$ did not change by more than $\pm 5 \%$ over a run of 200 trials (four blocks). This practice criterion was used in all conditions and $\mathrm{d}^{\prime}$ was computed by using the single-interval d' table from Swets (1964). The psychometric function for each condition was fit by a straight line on the coordinates $d^{\prime}$ vs. $\Delta f$. The threshold values of $\Delta f$ were chosen at a $\mathrm{d}^{\prime}$ of 1.0 in all conditions.

\section{Results and Discussion}

The two-point psychometric functions are shown in Table 1. As can be seen, the introduction of the backward interference tone in the diotic condition increased the value of $\Delta \mathrm{f}$ required for $\mathrm{a}^{\prime}=1.0$ approximately 6 times over that required when there was not an interference tone (NI condition). The three dichotic backward interference conditions all produced approximately the same value of $\Delta \mathrm{f}$ required for $\mathrm{a}^{\prime} \mathrm{d}^{\prime}=1.0$. These threshold values of $\Delta \mathrm{f}$ were approximately 2.5 less than those required in the diotic backward interference cases on approximately 2.4 times those required when there was not an interference tone.

Thus, dichotic presentation of the test and interference tones can improve the frequency discriminability of the test tone in the single-interval backward interference task, but the improvement is not such that discrimination is the same as when there is no interference tone. That is, there is still backward interference of frequency discrimination in the single-interval dichotic cases. The final observation to be made is that the type of dichotic presentation does not appear to change the amount of interference.

The values of threshold $\Delta \mathrm{f}$ obtained in the NI and ToSo conditions compare favorably with those obtained by Yost, Berg, and Thomas (1976) for the similar stimulus and procedural conditions. Only the $T_{L} I_{R}$ dichotic condition has been tested previously. The experiment of Cudahy and Leshowitz (1974) is most similar to ours, and they measured a smaller amount of backward interference than we did in the $T_{L} I_{R}$ condition. However, in the Cudahy and Leshowitz (1974) study, the test tones varied in frequency around $800 \mathrm{~Hz}$ while the interference tone was at $900 \mathrm{~Hz}$. Thus, the $100-\mathrm{Hz}$, or more, separation between test and interference tone frequency might explain the smaller amount of backward interference obtained in their experiment. Other studies employing the $T_{L} T_{R}$ backward interference condition (Leshowitz \& Cudahy, 1973; Massaro, 1970; Sparks, 1976) have obtained more or less amounts of

Table 1

Values of Two-Point Psychometric Functions for Backward Interference of Diotic vs. Dichotic Frequency Recognition

\begin{tabular}{|c|c|c|c|c|c|c|c|c|c|c|}
\hline & \multicolumn{5}{|c|}{ Subject 1} & \multicolumn{5}{|c|}{ Subject 2} \\
\hline & $\begin{array}{l}\Delta \mathrm{f}_{\mathbf{H}} \\
(\mathrm{Hz})\end{array}$ & $\mathrm{d}^{\prime} \mathbf{H}$ & $\underset{(\mathbf{H z})}{\Delta \mathbf{f}_{\mathbf{L}}}$ & $\mathrm{d}^{\prime} \mathrm{L}$ & $\begin{array}{c}\Delta f, d^{\prime}=1.0^{*} \\
(\mathrm{~Hz})\end{array}$ & $\begin{array}{l}\Delta \mathbf{f}_{\mathbf{H}} \\
(\mathbf{H z})\end{array}$ & $\mathrm{d}^{\prime} \mathbf{H}$ & $\begin{array}{l}\Delta \mathbf{f}_{\mathbf{L}} \\
(\mathbf{H z})\end{array}$ & $\mathrm{d}^{\prime} \mathbf{L}$ & $\begin{array}{c}\Delta \mathrm{f}, \mathrm{d}^{\prime}=1.0^{*} \\
(\mathrm{~Hz})\end{array}$ \\
\hline $\begin{array}{l}\text { NI (no interference) } \\
\text { Tolo (diotic) } \\
\text { TL I }_{\mathbf{R}} \text { (dichotic) } \\
\text { Tmlo (dichotic) } \\
\text { Tmlo (dichotic) }\end{array}$ & $\begin{array}{r}20 \\
100 \\
50 \\
50 \\
50\end{array}$ & $\begin{array}{l}1.64 \\
1.79 \\
1.46 \\
1.42 \\
1.77\end{array}$ & $\begin{array}{l}10 \\
60 \\
16 \\
20 \\
14\end{array}$ & $\begin{array}{l}.90 \\
.61 \\
.72 \\
.65 \\
.45\end{array}$ & $\begin{array}{l}11.4 \\
73.2 \\
27.8 \\
30.6 \\
29.0\end{array}$ & $\begin{array}{l}20 \\
80 \\
40 \\
40 \\
40\end{array}$ & $\begin{array}{l}2.56 \\
2.35 \\
2.57 \\
2.43 \\
2.42\end{array}$ & $\begin{array}{l}10 \\
40 \\
16 \\
20 \\
20\end{array}$ & $\begin{array}{r}1.20 \\
.82 \\
.78 \\
.75 \\
.77\end{array}$ & $\begin{array}{r}8.5 \\
45.7 \\
18.9 \\
21.9 \\
21.8\end{array}$ \\
\hline
\end{tabular}

"Threshold value of $\Delta f$ derived from two-point function when $d^{\prime}=1.0$. 
backward interference of frequency discrimination than that obtained in the present study. However, differences in procedures, stimuli, and amount of subject training make any quantitative comparisons among the stlidies difficult, if not impossible.

As in the Yost, Berg, and Thomas (1976) study, as well as in that by Loeb and Holding (1975), the subjects in Experiment 1 required a great deal of practice before they reached the end of training. This was true for both the diotic (between 1,0002,000 trials of practice) and the dichotic conditions (between 800 and 1,200 trials for the $T_{L} I_{R}$ condition), although less practice time was required to learn the TmIo (between 600 and 800 trials) and the TrIo (400-800 trials) tasks once the $T_{L} I_{R}$ conditions had been investigated. Following testing on the three dichotic conditions, the diotic condition was retested and there was no change in performance from the results shown in Table 1 . The stability of the results can perhaps be emphasized by noting that Subject S2 completed all but the TrIo condition before becoming ill. Before she completed this condition, she participated in another backward interference experiment involving the diotic condition for 5 months. Immediately after completing that study, she was retested on the diotic and other dichotic ( $T_{L} I_{R}$ and $\left.T m I o\right)$ conditions, and the results were essentially the same as those shown in Table 1.

In order to determine if any changes in frequency discrimination would occur in the dichotic case for forward interference (test tone occurring after the termination of the interfering tone) or at longer temporal separations in the backward interference task between the test and interference tones, a second experiment was conducted with two new observers using the single-interval, ToSo diotic and TrSo dichotic conditions. We also measured the frequency discriminability of both a To and a T $\pi$ test tone in the absence of an interfering tone.

\section{EXPERIMENT 2}

\section{Method}

Subjects. Two new subjects (S3, S4) were hired under the same conditions as described in Experiment 1.

Stimuli. The identical Tolo, Trlo, and NI conditions as described in Experiment 1. The frequency discrimination of the $\mathrm{T}$ test tone without the interference tone was also tested (the NI, $T_{\pi}$ condition). In addition, the Tolo and $T \pi I_{0}$ conditions were tested when the test tone ended $100 \mathrm{msec}$ (instead of $5 \mathrm{msec}$ as in Experiment 1) before the interference tone began (the BI-100 msec condition as compared to the Bl-5-msec condition of Experiment 1). A forward interference condition was used in the Tolo and $\mathrm{T} \pi \mathrm{lo}$ conditions in which the interference tone ended $5 \mathrm{msec}$ before the test tone began (FI-5-msec condition). All other stimulus parameters were the same as in Experiment 1.

Procedures. All procedures were the same as in Experiment 1.

\section{Results and Discussion}

The results are shown in Table 2 as the values of
$\Delta \mathrm{f}$ required for $\mathrm{a} \mathrm{d}^{\prime}=1.0$ obtained for each subject in the various conditions. The results for the backward interference, 5-msec conditions are in agreement with those of Experiment 1, although Subjects S3 and S4 had somewhat lower values of threshold $\Delta \mathrm{fs}$ than did Subjects $\mathrm{S} 1$ and $\mathrm{S} 2$. Also, there was essentially no difference in frequency discrimination between a diotic test tone (To) and a dichotic test tone $(\mathrm{T} \pi)$ presented without an interfering tone. There was no significant backward interference of frequency discrimination at $100 \mathrm{msec}$ or forward interference of frequency discrimination in either the diotic or dichotic conditions.

As in Experiment 1, considerable practice was required in the backward interference conditions before Subjects S3 and S4 achieved the criterion for ending practice. More practice was required in the diotic cases $(1,200-1,400$ trials) than in the dichotic cases (800-1,100 trials). Little practice was required in the forward interference conditions (200-400 trials).

\section{GENERAL DISCUSSION AND CONCLUSIONS}

These data show that there is backward interference of frequency discrimination in a singleinterval task in which the test and interfering tone are presented either diotically or dichotically. The amount of backward interference is much less in the dichotic cases than in the diotic case, and the amount of backward interference is independent of the type of dichotic presentation. Interference of frequency discrimination appears only when the test tone is close in time to the backward interference tone and does not occur in a forward interference condition.

We would not expect any backward interference in the dichotic conditions when other psychophysical tasks are used, since Yost, Berg, and Thomas (1976)

Table 2

Diotic vs. Dichotic Frequency Recognition as a Function of Temporal Position of Interference

\begin{tabular}{|c|c|c|}
\hline & Subject 3 & Subject 4 \\
\hline & $\Delta \mathrm{f} @ \mathrm{~d}^{\prime}=1.0^{\mathrm{a}}$ & $\Delta \mathrm{f} @ \mathrm{~d}^{\prime}=1.0^{\mathrm{a}}$ \\
\hline $\begin{array}{l}\text { NI }(\mathrm{To})^{b} \\
\text { NI }(\mathrm{T} \pi)\end{array}$ & $\begin{array}{l}5.1 \\
7.7\end{array}$ & $\begin{array}{l}9.2 \\
8.8\end{array}$ \\
\hline $\begin{array}{l}\text { Tolo, B 1-5c } \\
\text { Trlo, B 1-5 }\end{array}$ & $\begin{array}{l}40.0 \\
17.8\end{array}$ & $\begin{array}{l}38.9 \\
15.6\end{array}$ \\
\hline $\begin{array}{l}\text { Tolo, B 1-100 } \\
\text { Tilo, B1-100 }\end{array}$ & $\begin{array}{l}5.2 \\
5.1\end{array}$ & $\begin{array}{l}11.9 \\
10.9\end{array}$ \\
\hline $\begin{array}{l}\text { Tolo, F1-5 } \\
\text { T } \pi 10, F 1-5\end{array}$ & $\begin{array}{l}4.5 \\
4.4\end{array}$ & $\begin{array}{l}7.8 \\
9.8\end{array}$ \\
\hline
\end{tabular}

Note-The first member of each pair listed is diotic, the second, dichotic. All values are given in hertz. ${ }^{a}$ Threshold value of $\Delta f$ derived from two-point function when $d^{\prime}=1.0 .{ }^{b} N I-n o$ interference. ${ }^{c} B 1-5$-interfering tone followed test tone by 5 msec.

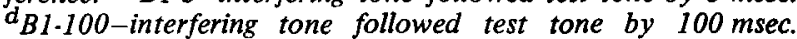
${ }^{e}$ F1-5-interfering tone preceded test tone by $5 \mathrm{msec}$. 
found little if any backward interference in the diotic case for procedures other than the single-interval procedure. However, if nontrained observers were used and/or if stimulus uncertainty were introduced into these tasks, then one might be able to obtain backward interference in both diotic and dichotic listening conditions.

To the extent that the preperceptual model of Massaro's (1972) can account for the data obtained in the backward interference single interval condition (see Yost, Berg, \& Thomas, 1976, for discussion of the preperceptual procedure), the results of the present study imply that to some extent the effect is central. However, since there is a clear difference in the amount of backward interference between the diotic and dichotic conditions, there also must be some peripheral component to the backward interference of frequency discrimination.

In conditions in which test and interfering stimuli are presented simultaneously, improvement in discrimination (including frequency intensity, duration, and speech discrimination or recognition) occurs for dichotic presentation over that obtained for diotic presentations only near threshold intensities of the test tone (see Green \& Yost, 1975, for a review). In the masking-level difference (MLD) literature (see Green \& Yost, 1975), a dichotic test tone can be detected at lower masked thresholds than can a diotic test tone, but dichotic discrimination thresholds are not smaller than diotic discrimination thresholds except when the test tones are near masked thresholds in the diotic conditions. The results from the present temporal interference experiment appear to be an exception to this rule. The test tone is clearly audible (well above masked threshold) in both the diotic and dichotic conditions, and yet dichotic frequency discrimination thresholds are smaller than diotic thresholds. Although this implies an improvement in discrimination caused by binaural listening, the "improvement" is due to the fact that discrimination in the diotic case is poor.

Thus, the results of this experiment suggest that the pitch of the test tone in the single-interval, back: ward interference task is easier to identify in dichotic conditions than in diotic conditions. The subjective reports of our subjects agree with this generalization in that they reported that the test tone was easier "to separate" from the interference tone in the dichotic conditions. This can perhaps be explained by the fact that in each dichotic condition the test tone occupies a different lateral (in the head) location than the interfering tone, since each was presented with dissimilar interaural differences (interaural differences of time or intensity). Thus, the spatial separation via the binaural system of the two tones allows for easier identification of the pitch of the test tone. To some extent, this might be analogous to the "cocktail party" effect, in which binaural processing allows one to attend to or identify a particular "voice" (test tone) from a background of competing "voices" (interfering tone). That is, in the singleinterval, backward interference tasks, the test tone can be easily detected in the diotic and dichotic conditions, but its pitch is more difficult to identify in the diotic than in the dichotic condition. The dichotic condition separates the test and interference stimuli in lateral space which might make it easier for the subject to identify the pitch of the test tone. If a similar process occurs in the cocktail party effect, then the ability to recognize a voice is degraded when the voice and an interfering stimuli are temporally proximal. This interference can be reduced if the voice to be identified occupies a different spatial location than the interfering voices or stimuli.

One might argue that the pitch of the test tone was easier to hear in the dichotic cases, since there would be less masking (partial masking) for the dichotic test tones than for the diotic test tone. Several aspects of binaural listening and backward interference argue against this explanation. First, Townsend and Goldstein (1972) have shown that when both diotic and dichotic signals have intensities well above masked thresholds (as the test tone is in the backward interference conditions) there is no difference in their judged loudnesses. Thus, the diotic and dichotic test tones are probably equal in loudness, which would be inconsistent with an asymmetry in the amount of partial masking. Second, as stated earlier, discrimination differences have not been obtained for dichotic and diotic test tones presented in simultaneous masking experiments. Third, recent experiments (to be published) in our laboratories have shown that backward interference occurs over a large range of relative intensities of loudnesses of the test and interference tones. Finally, the masking-level difference literature shows that the masked threshold for the test tone in the $T_{L} I_{R}$ condition should be lower than that in the TrIo condition, which, in turn, would be lower than that in the TmIo condition. Yet, frequency discrimination thresholds for these three dichotic conditions are essentially the same.

Therefore, the data of the present study imply that dichotic presentation of test and interference tones in a single-interval backward interference condition can reduce the threshold for frequency discrimination below that obtained with diotic presentations of test and interference tones. The dichotic frequency discrimination thresholds are still greater than those obtained for the test tone presented without a backward interfering tone. The improvement in frequency discrimination achieved with dichotic presentation might be a result of the spatial separation of the test and interference tones. 


\section{REFERENCES}

Cudahy, E.. \& Leshowitz, B. Effects of a contralateral interference tone on auditory recognition. Perception \& Psychophysics, 1974, 15, 16-21.

Green, D. M., \& Swets. J. A. Signal detection theory and psychophysics. New York: Krieger, 1974.

Green, D. M..\& Yost, W. A. Binautal analysis. In W. D. Keidel \& W. D. Neff (Eds.), Handbook of sensory physiology (Vol. 2). New York: Springer-Verlag, 1975.

LEShowitz, B.. \& CUdAHY, E. Frequency discrimination in the presence of another tone. Joumal of the Acoustical Society of America, 1973, 54, 883-887.

LOEB, M., \& Holding, D. H. Backward interference by tones or noise in pitch perception as a function of practice. Perception \& Psychophysics, 1975, 18, 225-229.

MASSARO, D. W. Pre-perceptual auditory images. Journal of Experimental Psychology, 1970, 85, 411-417.

Massaro, D. W. Pre-perceptual images, processing time, and perceptual units in auditory perception. Psychological Review, 1972, 79, 124.145.

Massaro, D. W. Experimental psychology and information processing. Chicago: Rand-McNally, 1975.

SPARKS, O. W. Backward pitch-recognition interference. Journal of the Acoustical Society of America, 1976, 60, S49(A).

SwETs, J. A. (Ed.) Signal detection and recognition by human observers: Contemporary readings. New York: Wiley, 1964.

Townsend, T. H., \& Goldstein, D. P. Suprathreshold binaural unmasking. Journal of the Acoustical Society of America, 1972, 51, 621.624.
Yost, W. A., Berg, K., \& Thomas, G. B. Frequency recognition in temporal interference tasks: A comparison among four psychophysical procedures. Perception \& Psychophysics, 1976. 20. 353-359.

\section{NOTES}

1. The nomenclature used in the present study is similar to that used to describe the diotic and dichotic conditions in studies of binaural masking (see Green \& Yost, 1975) if one uses S (signal) instead of $T$ and $M$ or $N$ (masker or noise) instead of $1 . S, M$, or $\mathrm{N}$ were not used, since they are used in connection with detection experiments involving masking. Since the test tone in the present experiment is not masked (i.e., it is well above threshold) but rather might have its frequency threshold shifted, we prefer not to refer to our experiment as masking but rather as interference. Thus, to avoid the implication of masking, we have used the symbols $\mathrm{T}$ (test tone) and I (interference tone).

2. Pilot experiments convinced us that there were no significant differences between presenting the tones in the $T_{L} I_{R}$ vs. $T_{R} I_{L}$ dichotic configurations, so we always used the $T_{L} I_{R}$ configuration. In the Tmlo condition, the test tone was again presented only to the left ear.

(Received for publication February 4, 1977; revision accepted June 12, 1977.) 